\title{
Daam1 activates RhoA to regulate Wnt5a-induced glioblastoma cell invasion
}

\author{
GUIYANG LIU $^{1 *}$, TING YAN $^{2 *}$, XIAORONG LI ${ }^{1}$, JIANHUI SUN $^{1}$, BO ZHANG $^{1}$, \\ HONGJIE WANG ${ }^{1}$ and YICHAO $\mathrm{ZHU}^{3,4}$ \\ ${ }^{1}$ Department of Neurosurgery, Jinan Fourth People's Hospital, Jinan, Shandong 250031; \\ ${ }^{2}$ Safety Assessment and Research Center for Drug, Pesticide and Veterinary Drugs of Jiangsu Province, \\ School of Public Health, ${ }^{3}$ Department of Physiology and ${ }^{4}$ State Key Laboratory of Reproductive Medicine, \\ Nanjing Medical University, Nanjing, Jiangsu 211166, P.R. China
}

Received April 13, 2017; Accepted September 27, 2017

DOI: $10.3892 /$ or.2017.6124

\begin{abstract}
The signaling pathway of dishevelled-associated activator of morphogenesis 1 (Daam1) triggered by Wnt5a drives cell movement and migration during breast cancer metastasis. However, Wnt5a signaling in glioblastoma progression remains poorly defined. Wnt5a expression and activations of RhoA, Cdc42, and Racl were detected in human glioblastoma tissues by using ELISA assays and small G-protein activation assays, respectively. The cell invasion rate and Daam1 activation of glioblastoma U251 and T98MG cells were determined by cell invasion assays and pull-down assays, respectively. According to our experiments, Wnt5a expression and RhoA activation were upregulated in invasive glioblastoma tissues, with a significant positive correlation between them. Wnt5a activated Daam1 and RhoA, and subsequently promoted the invasion of glioblastoma U251 and T98MG cells. This process was abolished by secreted frizzled-related protein 2 (sFRP2), an antagonist that directly binds to Wnt5a. Specific small interfering RNA (siRNA) targeting Daam1 markedly inhibited Wnt5a-induced RhoA activation, stress fiber formation and glioblastoma cell invasion. CCG-1423, a RhoA inhibitor, decreased Wnt5a-induced stress fiber formation and glioblastoma cell invasion. Finally, siRNA targeting Daam1 or CCG-1423 treatment did not alter the cell proliferation of glioblastoma U251 and T98MG cells. We thus concluded that
\end{abstract}

Correspondence to: Professor Hongjie Wang, Department of Neurosurgery, Jinan Fourth People's Hospital, 50 Shifan Road, Jinan, Shandong 250031, P.R. China

E-mail: wanghongdoctor@163.com

Dr Yichao Zhu, Department of Physiology, Nanjing Medical University, 101 Longmian Road, Nanjing, Jiangsu 211166, P.R. China E-mail: zhuyichao@njmu.edu.cn

*Contributed equally

Key words: Wnt5a, Daam1, RhoA, invasion, glioblastoma
Wnt5a promoted glioblastoma cell invasion via Daam1/RhoA signaling pathway.

\section{Introduction}

Glioblastoma, also known as glioblastoma multiforme (GBM), is the most aggressive primary tumor of the brain (1-3). Despite advances in surgical technology and adjuvant treatment for glioblastoma, this tumor remains the most lethal disease worldwide $(2,3)$. The molecular mechanisms underlying the invasion and metastasis of glioblastoma are still unknown.

Formerly, Wnts have been divided into two classes: those that signal through canonical ( $\beta$-catenin dependent) pathway and those that signal through non-canonical ( $\beta$-catenin independent) pathway (4-6). Wnt signaling participates in the development of embryo and pathological processes, including tumorigenesis and metastasis (7). A homeobox transcription factor, MSX1 was able to inhibit the $\mathrm{Wnt} / \beta$-catenin signaling pathway and suppress $W n t / \beta$-catenin-induced migration and invasion of cultured glioblastoma cells (8). Inhibition of the Wnt/ $\beta$-catenin pathway significantly abrogated the invasion effects of irradiation, indicating a pivotal role of the Wnt/ $\beta$-catenin pathway in ionizing radiation-induced invasion of U87 cells (9).

Wnt5a is classified as a non-transforming Wnt family member that plays complex roles in cancer initiation and metastasis (10-12). Wnt5a activates small Rho-GTPases and regulates the cytoskeletal architecture and cellular polarity during development (13). Wnt5a signaling is a regulator in the proliferation of human glioma cells (14). However, very few studies have reported on the role of Wnt5a in glioblastoma cell invasion and migration $(15,16)$. In the present study, we propose for the first time that Wnt5a promotes the invasion of glioblastoma cells and is upregulated in invasive glioblastoma tissues. Moreover, the mechanisms whereby the Wnt5a/Daam1/RhoA signaling pathway regulates glioblastoma cell invasion are described.

\section{Materials and methods}

Clinical samples. Tissue samples of nine glioblastoma patients from the Jinan Fourth People's Hospital from 2015 to 2016 were recruited in the present study. All patients underwent surgical 
resection of the glioblastoma with the intention of maximally resecting the tumor. Glioblastoma tissues were frozen in liquid nitrogen or fixed in $10 \%$ formalin and embedded in paraffin wax. Slices were stained with hematoxylin and eosin (H\&E) for light microscopy. The diagnosis of glioblastoma was based on the World Health Organization (WHO) 2007 and 2016 histopathologic criteria: the invasive phenotype showing an infiltrative astrocytic neoplasm with high proliferative activity and microvascular proliferation, necrosis or both $(17,18)$. The non-invasive glioblastoma was thus called when the tumor at the anatomical site began to progress and further yielded a cancerous mass without the invasive phenotype aforementioned. All glioblastoma tissues with high tumor cell density were histopathologically confirmed by two pathologists prior to ELISA and small G-protein activation assay. Ethical approval of the study (no. LL-20140005) was granted by the Clinical Research Ethics Committee, Jinan Fourth People's Hospital. Written informed consent was obtained from each participant.

Cells and small interfering RNA (siRNA) transfection. U87MG, U251 and T98MG are the most used cell lines for research on human glioblastoma. The original U87MG cell line was established in Uppsala University almost 50 years ago (19). However, Allen et al used short tandem repeat (STR) genotyping to screen out the DNA profile of U87MG. Different from that of the original cells, this friendly profile of U87MG is thought to have an unknown origin (20). Thus, two cell lines (U251 and T98MG) were used in this experiment. Human glioblastoma U251 or T98MG cell lines were purchased from the Cell Bank of Shanghai (Shanghai, China) and were grown in Eagle's Minimum Essential Medium (EMEM; HyClone, Thermo Scientific, Waltham, MA, USA) supplemented with $10 \%$ (v/v) fetal bovine serum (FBS), $2 \mathrm{mmol} / \mathrm{l} \mathrm{L}$-glutamine and $100 \mathrm{IU} / \mathrm{ml}$ penicillin, $100 \mu \mathrm{g}$ streptomycin, $1 \mathrm{mmol} / 1$ sodium pyruvate and non-essential amino acids (HyClone) in a humidified incubator at $37^{\circ} \mathrm{C}$ with $5 \% \mathrm{CO}_{2}$ and $95 \%$ humidity. The cells were seeded in 6-well plates (Costar, Corning, NY, USA) and cultured to $80 \%$ confluence, and then transiently transfected with siRNA against Daam1 (21) using Lipofectamine 2000 reagent (Invitrogen, Carlsbad, CA, USA) in serum-free Opti-MEM according to the manufacturer's instructions. The cells were switched to fresh medium containing $10 \%$ FBS $6 \mathrm{~h}$ after the transfection and cultured for $48 \mathrm{~h}$. The cells transfected with Daam1-siRNA were used for analyzing Rho activation and cell invasion.

ELISA. The glioblastoma tissues were grinded in liquid nitrogen. Equal weights of total tissue debris were dissolved in ice-cold phosphate-buffered saline (PBS) buffer. The experiments were then performed according to the manufacturer's protocol of the Wnt5a ELISA kit (CusaBio, Wuhan, China). The concentration of each glioblastoma tissue was calculated based on the concentration curve of the Wnt5a standard samples.

Cell invasion assays. Cell invasion was assessed in modified Boyden chambers (Costar). Two chambers were separated by a polycarbonate membrane (pore diameter, $8.0 \mu \mathrm{m}$ ). Boyden chamber wells were coated with Matrigel (BD Biosciences,

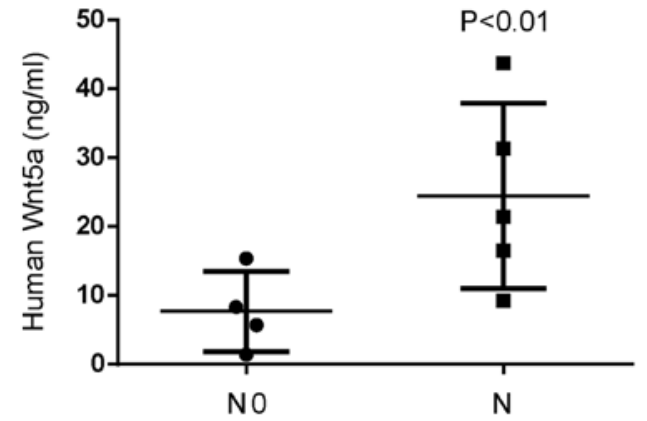

Figure 1. Wnt5a is upregulated in invasive glioblastoma tissues. The expression level of Wnt5a was highly expressed in invasive glioblastoma tissues $(n=5)$ when compared to that in non-invasive glioblastoma tissues $(n=4)$. N0, non-invasive glioblastoma; $\mathrm{N}$, invasive glioblastoma.

Franklin Lakes, NJ, USA) for $30 \mathrm{~min}$ at $37^{\circ} \mathrm{C}$. U251 or T98MG cells treated with CCG-1423 (Selleck, Houston, TX, USA) were added to wells with a membrane placed in the bottom. Medium containing recombinant Wnt5a (rWnt5a) was added to the upper and lower compartment of the Boyden chamber. The cells were allowed to invade for $6 \mathrm{~h}$ at $37^{\circ} \mathrm{C}$ in this assay. Thereafter, the medium was discarded, stationary cells were removed with a cotton-tipped applicator, and the membranes were cut out of the chamber and stained with $0.5 \%$ crystal violet. The response was evaluated on a light microscope by counting the number of cells that had invaded into the Matrigel and membrane.

Small G-protein activation assay. For RhoA, Cdc 42 and Rac1 activation assays, the glioblastoma tissues were grinded in liquid nitrogen. Equal weights of total tissue debris were dissolved in ice-cold PBS buffer. Glioblastoma cells were seeded into 6-well plates and transfected with Daam1-siRNA or treated with sFRP2 (R\&D Systems, Minneapolis, MN, USA). The experiments were then performed according to the manufacturer's protocol (Cytoskeleton Inc., Denver, CO, USA). The activation of RhoA, Cdc42 and Racl was normalized to the NC control group.

Western blotting. Subconfluent cells were washed twice with PBS, and then lysed with ice-cold RIPA lysis buffer (Beyotime Biotechnology, Nantong, China). The lysates were then clarified by centrifugation at $12,000 \mathrm{x} \mathrm{g}$ for $20 \mathrm{~min}$ at $4^{\circ} \mathrm{C}$. The protein extracts were separated by $8 \%$ sodium dodecyl sulfate-polyacrylamide gel electrophoresis (SDS-PAGE). The immunoblotting procedure was performed as previously described (22), and the following antibodies were used: anti-GAPDH (Sigma, St. Louis, MO, USA), anti-Daam1 (Santa Cruz Biotechnology, Santa Cruz, CA, USA) antibodies. Protein bands were detected by incubation with horseradish peroxidase-conjugated antibodies and visualized with enhanced chemiluminescence (ECL) reagent (Thermo Scientific, Rockford, IL, USA).

Pull-down assays. For the detection of active Daam1, GST-RhoA beads were incubated with $0.1 \mathrm{mmol} / 1 \mathrm{GTP} \gamma \mathrm{S}$ (Sigma) at $30^{\circ} \mathrm{C}$ for $15 \mathrm{~min}$ with constant agitation. Equal volumes of total cellular protein were incubated with GST-RhoA beads captured on MagneGST Glutathione 
A

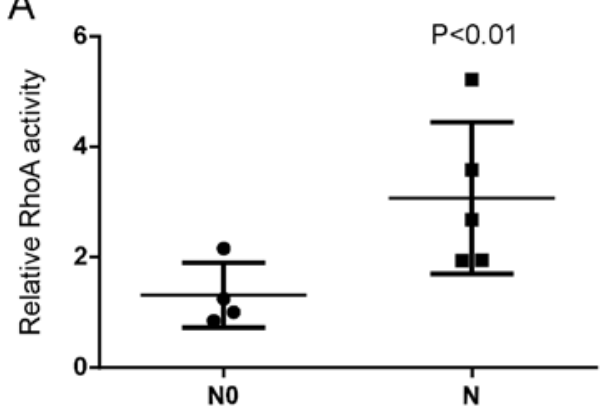

B
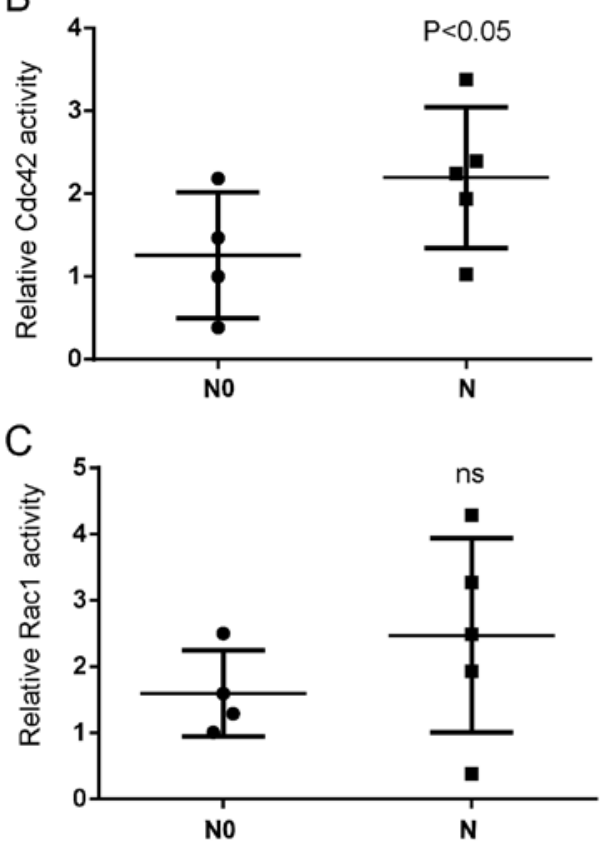

D

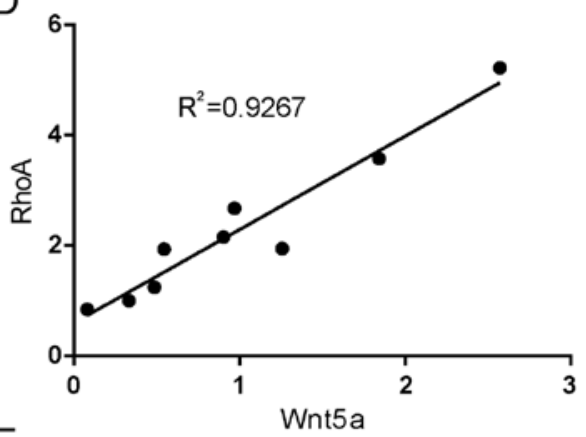

E

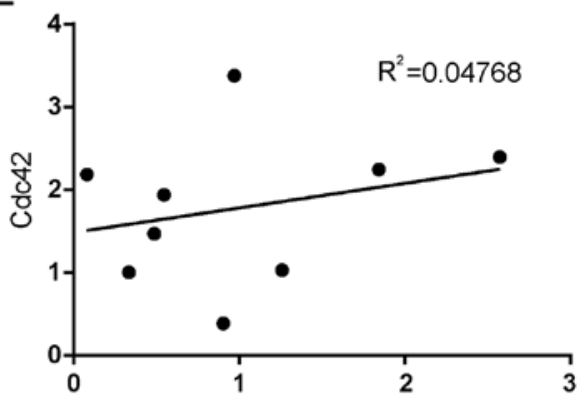

F

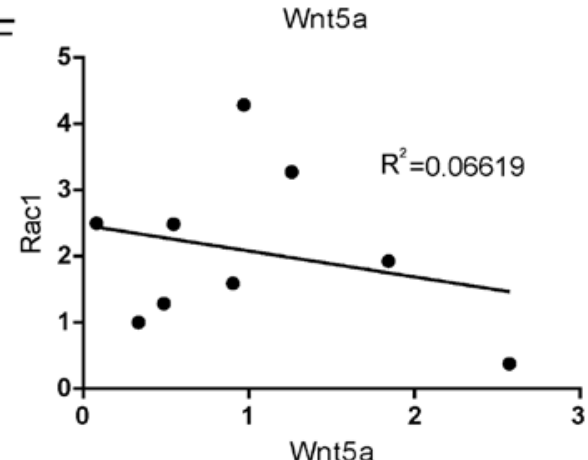

Figure 2. RhoA is activated and positively correlated with Wnt5a in invasive glioblastoma tissues. (A-C) The activation of (A) RhoA and (B) Cdc42 was highly expressed in invasive glioblastoma tissues $(n=5)$ when compared to that in non-invasive glioblastoma tissues ( $\mathrm{n}=4)$. $(\mathrm{C})$ The activations of Racl exhibited no significant difference between invasive glioblastoma and non-invasive glioblastoma tissues. N0, non-invasive glioblastoma; N, invasive glioblastoma; ns, no significance. (D-F) The correlation analysis in glioblastoma tissues was performed between the expression of Wnt5a and activation of RhoA, Cdc42 and Rac1. (D) The activation of RhoA was positively correlated with Wnt5a expression. Correlation coefficients $\left(\mathrm{R}^{2}\right)$ are shown in the panels.

Particles (Promega, Madison, WI, USA) at $4^{\circ} \mathrm{C}$ with constant rotation for $90 \mathrm{~min}$. The beads were washed three times with washing buffer $\left(4.2 \mathrm{mmol} / 1 \mathrm{Na}_{2} \mathrm{HPO}_{4}, 2 \mathrm{mmol} / 1 \mathrm{KH}_{2} \mathrm{PO}_{4}\right.$, $140 \mathrm{mmol} / \mathrm{l} \mathrm{NaCl}$ and $10 \mathrm{mmol} / \mathrm{KCl}, \mathrm{pH} \mathrm{7.2)}$. At the end of this period, the beads were captured using a magnet on a magnetic stand. After being washed three times with ice-cold buffer, the beads were resuspended in Laemmli buffer, boiled, and subjected to western blot analysis. SDS-PAGE and western blotting were performed using standard methods.

Actin cytoskeleton staining and immunofluorescence. Transfected cells were fixed in $4 \%$ paraformaldehyde in PBS for $20 \mathrm{~min}$, permeabilized in $0.2 \%$ Triton X-100 and blocked in PBS containing 1\% BSA for $1 \mathrm{~h}$ at room temperature. F-actin was stained with FITC-labeled phalloidin $(5 \mathrm{mg} / \mathrm{ml})$ (Beyotime Biotechnology) for $40 \mathrm{~min}$ at room temperature. After being washed with PBS, the coverslips were mounted on glass slides with DAPI Fluoromount-G (Southern Biotech, Birmingham, AL, USA). The images were acquired with a fluorescence microscope (Zeiss, LSM 710 system; Carl Zeiss, Jena, Germany).
Statistical analysis. The data were analyzed using Student's t-test with the SPSS statistical software package. All the results were expressed as the mean \pm SD. For all analyses a two-sided P-value of $<0.05$ was deemed statistically significant.

\section{Results}

Wnt5a and RhoA are upregulated in invasive glioblastoma tissues. In order to evaluate Wnt5a expression in invasive glioblastoma tissues, we assessed the expression of Wnt5a using ELISA assays in nine samples of glioblastoma. Wnt5a expression was higher in invasive glioblastoma tissues compared to that in non-invasive glioblastoma tissues, with the highest expression at $43.7 \mathrm{ng} / \mathrm{ml}$ (Fig. 1). We also assessed the activations of Rho GTPases in the same glioblastoma tissues and found that the activation of RhoA and Cdc 42 were higher in invasive glioblastoma tissues compared to that in non-invasive glioblastoma tissues (Fig. 2A and B). However, Rac1 activity had an insignificant increase in invasive glioblastoma tissues compared to non-invasive glioblastoma tissues (Fig. 2C). Moreover, the activation of RhoA was positively correlated 
A

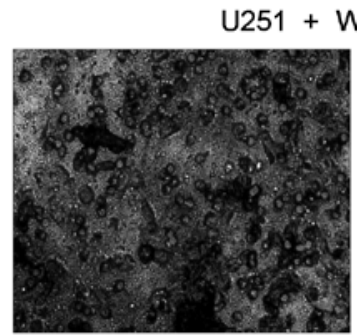

0

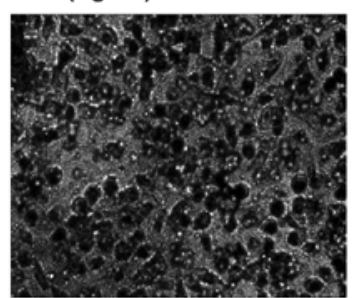

20
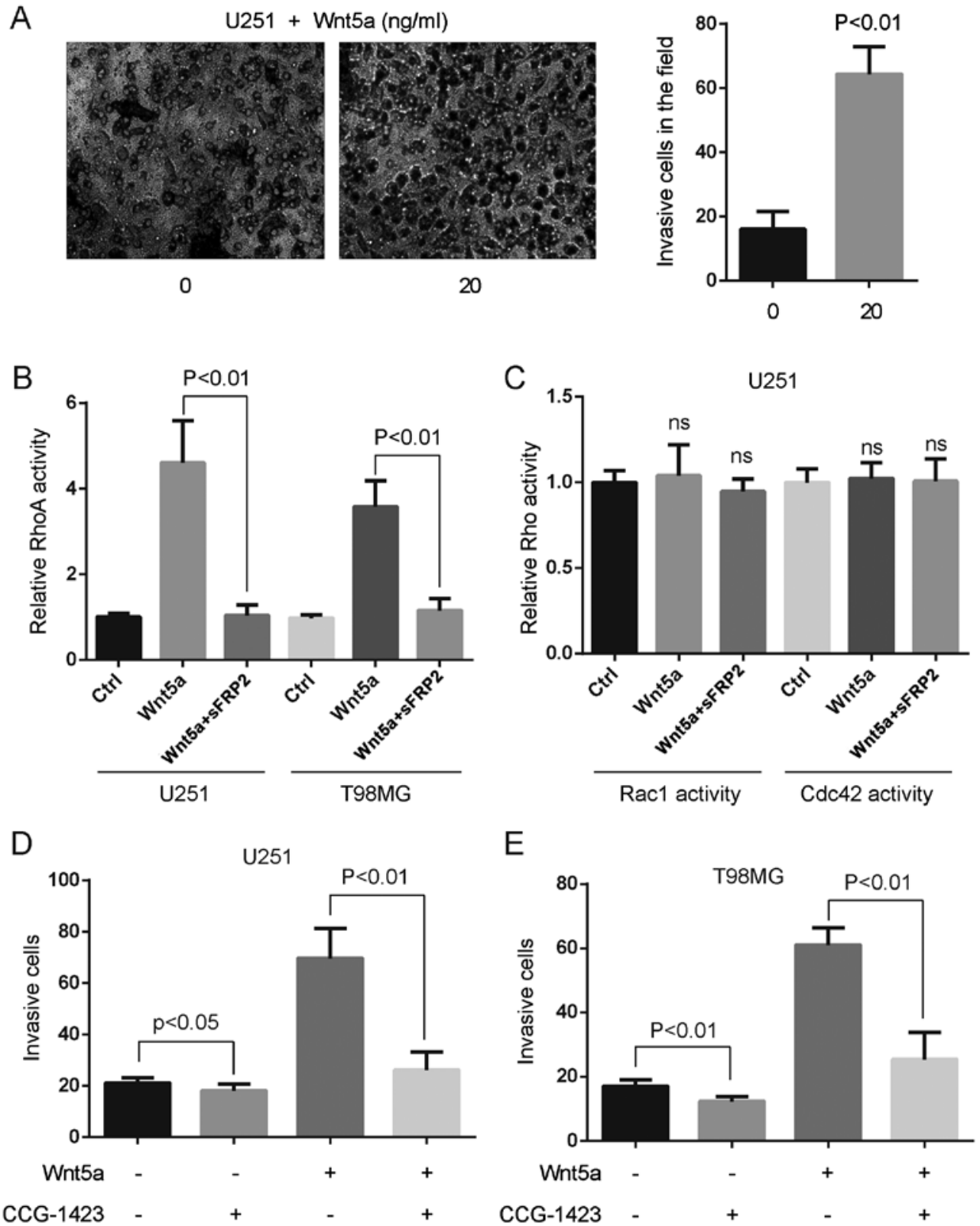

Figure 3. RhoA activation is required for Wnt5a-induced glioblastoma cell invasion. (A) U251 glioblastoma cells were treated with 20 ng/ml Wnt5a and allowed to invade for $6 \mathrm{~h}$. The rates of cell invasion were determined by Boyden chamber assays. The left panel shows the glioblastoma cells that had invaded onto the bottom surface. Magnification, x200. (B and C) RhoA activation, but not Rac1 and Cdc42 activation, was induced by Wnt5a, but blocked by sFRP2 treatment. Serum-starved U251 and T98MG cell monolayers were incubated with $20 \mathrm{ng} / \mathrm{ml} \mathrm{rWnt} 5 \mathrm{a}$ for $1 \mathrm{~h}$, or pre-treated with $100 \mathrm{ng} / \mathrm{ml}$ sfrp2 for $1 \mathrm{~h}$ and then incubated with $20 \mathrm{ng} / \mathrm{ml}$ recombinant Wnt5a (rWnt5a) for $1 \mathrm{~h}$. Cell lysates were assayed for small G-protein activation assay. ns, no significance. (D and E) Wnt5ainduced cell invasion was abolished by CCG-1423. U251 and T98MG cells were pre-incubated with RhoA-specific inhibitor CCG-1423 (10 ng/ml) for $1 \mathrm{~h}$, and then allowed to invade in Matrigels with $20 \mathrm{ng} / \mathrm{ml} \mathrm{rWnt5a}$ treatment for $6 \mathrm{~h}$. The cell invasion rate was determined by Boyden chamber assays.

with the expression of Wnt5a in glioblastoma tissues (Fig. 2D), while there was a weak correlation between the activation of Cdc42 or Racl and Wnt5a expression (Fig. 2E and F). These results indicated that Wnt5a and RhoA had tumor-promoting roles in glioblastoma invasion.

Wnt5a stimulates glioblastoma cell invasion in vitro. To assess the effect of Wnt5a on glioblastoma cell invasion, we treated U251 glioblastoma cells with $20 \mathrm{ng} / \mathrm{ml} \mathrm{rWnt} 5 \mathrm{a}$, and assessed the invasion rate using the Boyden chamber assay. An approximately threefold increase of cell invasion was observed in U251 cells

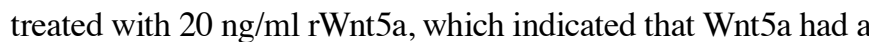
potent stimulatory effect on glioblastoma cell invasion (Fig. 3A).
RhoA activation participates in Wnt5a-induced glioblastoma cell invasion. We investigated whether RhoA activation was induced by Wnt5a in glioblastoma cells. Small G-protein assays revealed a significant increase of active RhoA after $20 \mathrm{ng} / \mathrm{ml}$ rWnt5a treatment (Fig. 3B). Pre-incubation of secreted frizzledrelated protein 2 (sFRP2), an antagonist that directly binds to Wnt5a (21), abolished rWnt5a-RhoA activity in U251 and T98MG cells (Fig. 3B). However, Wnt5a and/or sFRP2 treatment did not alter the activation of Racl and Cdc42 in U251 cells (Fig. 3C). To assess the effect of RhoA on glioblastoma cell invasion, we treated glioblastoma cells with $10 \mathrm{ng} / \mathrm{ml}$ RhoA-specific inhibitor CCG-1423 and $20 \mathrm{ng} / \mathrm{ml} \mathrm{rWnt} 5 \mathrm{a}$, and assessed the invasion rate using the Boyden chamber assay. We 
A

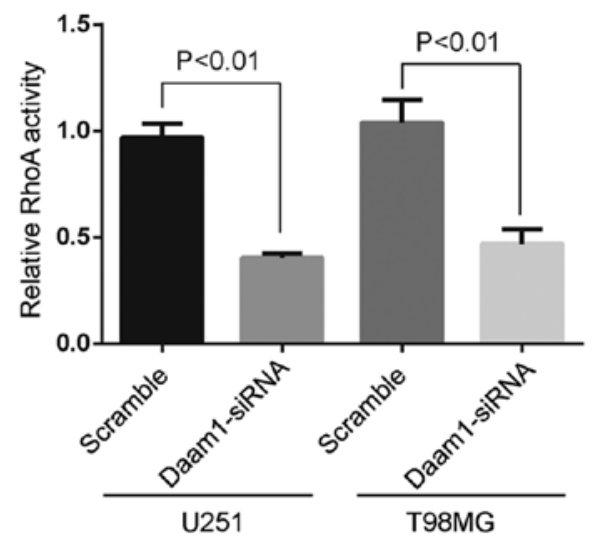

B

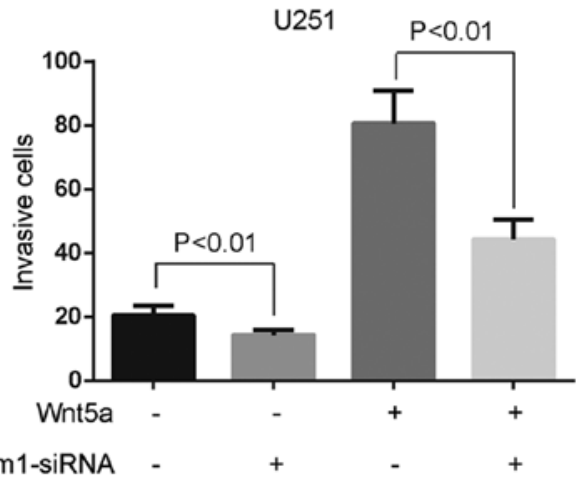

C

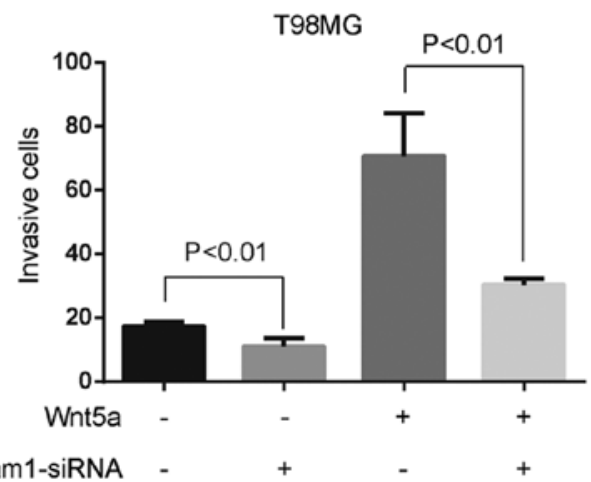

Figure 4. Dishevelled-associated activator of morphogenesis 1 (Daam1) is upstream of RhoA and its activation is essential for Wnt5a-induced glioblastoma cell invasion. (A) RhoA was a downstream target of Daam1. U251 and T98MG cells were transfected with Daam1 siRNA or scramble siRNA, and then incubated with $20 \mathrm{ng} / \mathrm{ml}$ recombinant Wnt5a (rWnt5a) for $1 \mathrm{~h}$. Cell lysates were assayed for small G-protein activation assay. (B and C) Wnt5ainduced cell invasion was abolished by Daam1 siRNA. U251 and T98MG cells were transfected with Daam1 siRNA or scramble siRNA, and then allowed to invade in Matrigels with $20 \mathrm{ng} / \mathrm{ml} \mathrm{rWnt} 5 \mathrm{a}$ treatment for $6 \mathrm{~h}$. The cell invasion rate was determined by Boyden chamber assays.

found that CCG-1423 blocked Wnt5a-induced glioblastoma cell invasion (Fig. 3D and E), indicating that RhoA activation was indispensable for Wnt5a-induced glioblastoma cell invasion.

Wnt5a induces glioblastoma cell invasion via Daaml activation. We examined whether Wnt5a-induced RhoA activation was regulated by Daam1 in glioblastoma cells Specific siRNA against Daam1 blocked Wnt5a-induced RhoA activation (Fig. 4A). Moreover, Daam1 siRNA was fully capable of retarding the invasion of U251 and T98MG
A
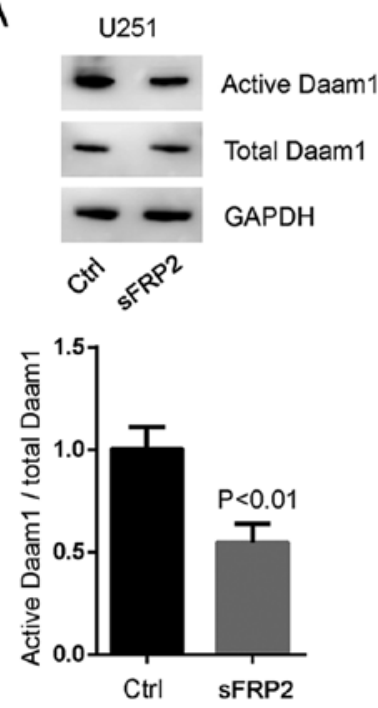

Figure 5. Dishevelled-associated activator of morphogenesis 1 (Daam1) activation is triggered by Wnt5a in glioblastoma cells. (A) Serum-starved U251 and (B) T98MG cell monolayers were pre-treated with $100 \mathrm{ng} / \mathrm{ml} \mathrm{sFRP2}$ for $1 \mathrm{~h}$, and then incubated with $20 \mathrm{ng} / \mathrm{ml}$ recombinant Wnt5a (rWnt5a) for $30 \mathrm{~min}$. Cell lysates were assayed for pull-down assays and western blotting. The ratios of active Daam1 and total Daam1 are displayed in the bottom panels. GAPDH was used as the internal control.

cells (Fig. 4B and C). To analyze the role of Wnt5a on Daam1 activation (a state allowing its interaction with RhoA), we blocked Wnt5a signaling with sFRP2 treatment. SFRP2 significantly inhibited Wnt5a-induced Daam1 activity in U251 and T98MG cells (Fig. 5A and B). These experiments demonstrated that RhoA was a downstream target of Wnt5a/Daam1 signaling in U251 and T98MG cells.

Wnt5a/Daam1/RhoA signaling sustains the formation of stress fibers. We performed fluorescent phalloidin staining to investigate the distribution pattern of filamentous actin (F-actin) in glioblastoma cells. Wnt5a treatment sustained the formation/maintenance of actin stress fibers in U251 cells (Fig. 6). In contrast, Daam1-siRNA or CCG-1423 treatment disrupted the formation of actin stress fibers in U251 cells (Fig. 6). Finally, neither Daam1-siRNA nor CCG-1423 treatment altered the cell proliferation of U251 and T98MG cells (Fig. 7A and B). Thus, the findings from the clinical and cellular biological assays indicated that RhoA activation required Daam1 activity to mediate Wnt5a-induced glioblastoma cell invasion (Fig. 7C).

\section{Discussion}

Wnt5a acts both as a suppressor and an inducer in different types of tumors (12,23-26). The Wnt/planar cell polarity (PCP) pathway triggered by Wnt5a activates small Rho-GTPases and reassembles the cytoskeletal architecture and cellular polarity during embryo development and pathological processes (27-29). In our previous studies, we found that Wnt5a stimulated the migration of breast cancer cells via Rho signaling pathways $(21,30)$. In gastric cancer, Wnt5a also enhanced the ability of cell mobility via RhoA signaling pathways (31). In the present study, we found that Wnt5a induced the invasion of U251 and T98MG glioblastoma cells. Moreover, 


\section{Merged}
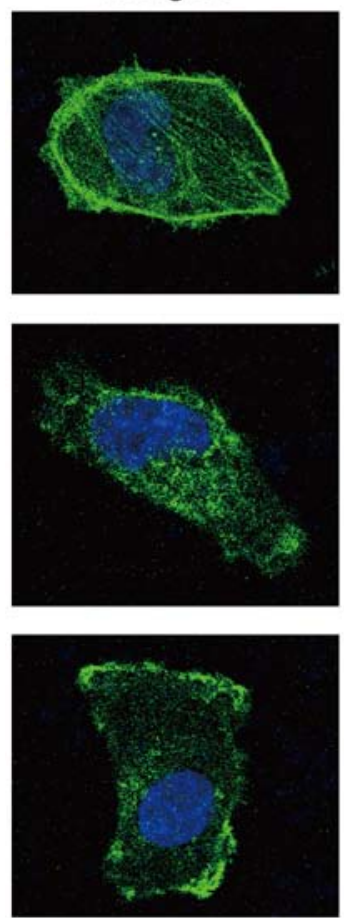

F-actin
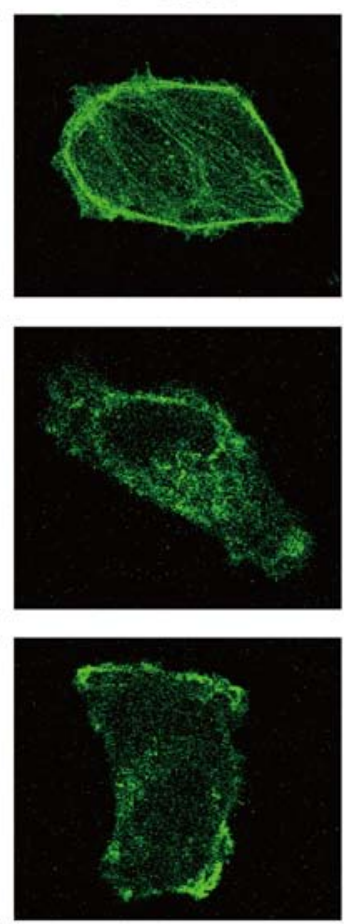

DAPI
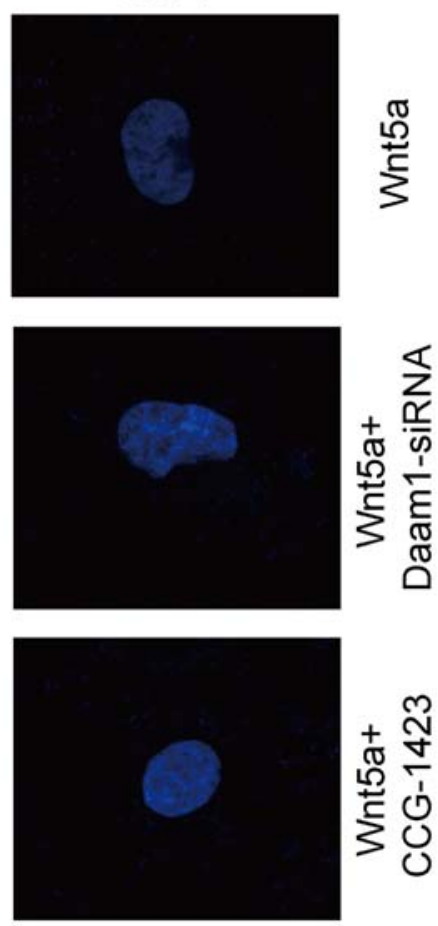

莫

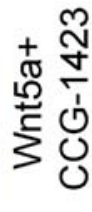

Figure 6. Daam1 and RhoA participates in the rearrangement of stress fibers in glioblastoma cells. Daam1 siRNA and CCG-1423 disrupted the formation of actin stress fibers in U251 cells. U251 cells were grown on coverslips, then transiently transfected with Daam1 siRNA or pre-treated with $10 \mathrm{ng} / \mathrm{ml}$ CCG-1423 for $1 \mathrm{~h}$. U251 cells were then incubated in culture medium containing $20 \mathrm{ng} / \mathrm{ml}$ recombinant Wnt5a (rWnt5a) for $4 \mathrm{~h}$. Subsequently, the cells were fixed and F-actin organization was analyzed by phalloidin staining. Magnification, $\mathrm{x} 400$.
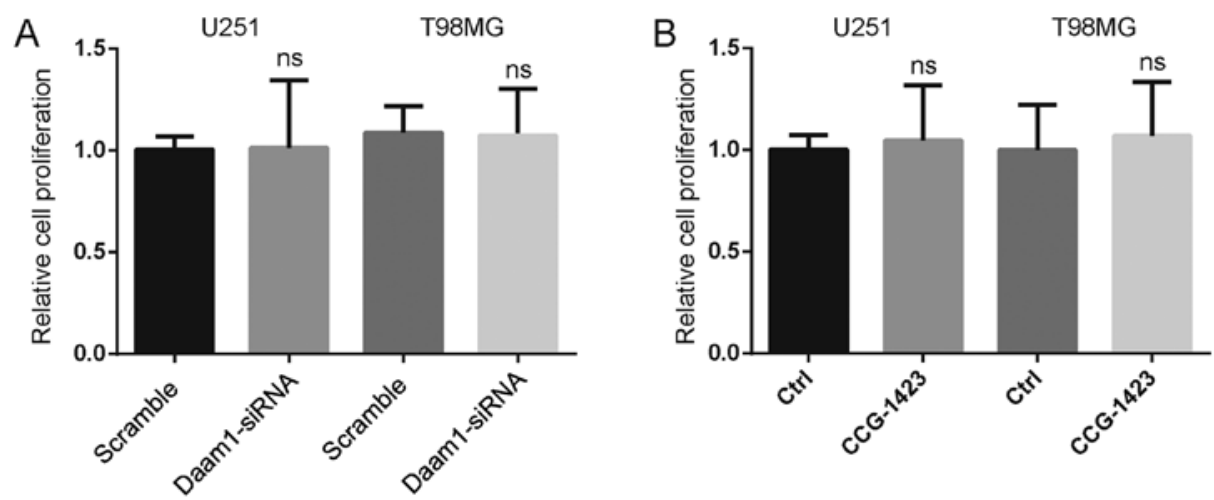

C

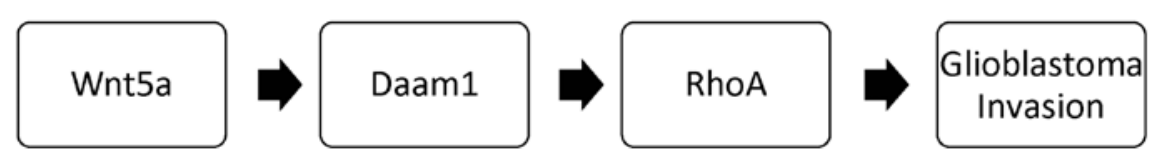

Figure 7. Daam1 and RhoA do not regulate the proliferation of glioblastoma cells. (A and B) U251 and T98MG cells were transfected with (A) Daam1 siRNA or (B) pre-treated with $10 \mathrm{ng} / \mathrm{ml} \mathrm{CCG-1423}$ for $1 \mathrm{~h}$, and then subjected to the cell proliferation assays. Daam1 siRNA and CCG-1423 did not alter the proliferation of glioblastoma cells; ns, no significance. (C) A schematic illustration of the regulation of RhoA in Wnt5a/Daam1 signaling to mediate cell glioblastoma invasion (see text for details).

Wnt5a was highly expressed in invasive glioblastoma tissues compared to that in non-invasive glioblastoma tissues. These results revealed that Wnt5a may accelerate glioblastoma invasion in vitro and in vivo and that blocking Wnt5a signaling may prevent glioblastoma metastasis in clinic. In addition, the overexpression of Wnt5a increased the proliferation of glioblastoma GBM-05 and U87MG cells, indicating that Wnt5a is a regulator in the proliferation of human glioblastoma (14).
This evidence demonstrated that Wnt5a acts as an inducer and promoter in glioblastoma tumorigenesis and metastasis.

With the help of small G-protein promoting cellular migration, Wnt5a can remodel the cellular cytoskeleton of melanoma cells (11). In the present study, Wnt5a promoted glioblastoma cell invasion by activating the Daam1/RhoA signaling pathway. In clinical samples, Wnt5a and RhoA are upregulated in invasive glioblastoma tissues, with a significant positive correlation 
between them. Studies with much larger samples may provide statistical power to validate the role of Wnt5a and RhoA in glioblastoma. Our previous study revealed that Racl activation was increased by Wnt5a and stimulated the cell migration of MCF-7 breast cancer cells (30). Unfortunately, Wnt5a did not alter the activation of Racl and Cdc42 in U251 glioblastoma cells in the present study. These results reveal the specificity of elevated RhoA activation in glioblastoma invasion.

Containing multiple regulatory domains, Daam1 exists in an auto-inhibited state through intramolecular interaction in unstimulated cells $(10,32)$. Our results showed that sFRP2, an antagonist that directly binds to Wnt5a, markedly blocked the activity of Wnt5a-induced Daam1 in glioblastoma cells. Knockdown of Daam1 expression via siRNA transfection inhibited RhoA activation and the cell invasion stimulated by Wnt5a in U251 and T98MG cells. Furthermore, we tested the reestablishment of stress fibers in Daam1-knockdown or RhoA-blocked cells. The decrease of RhoA activity, formation of stress fibers and cell invasion in Daam1-knockdown cells indicated that Daam1 may be required for the activation of RhoA after Wnt5a treatment in glioblastoma. Thus, these results clearly demonstrated that Daam1/RhoA signaling under Wnt5a stimulation participated in the invasion of glioblastoma and that retarding them may prevent glioblastoma metastasis in clinic.

Soluble frizzled-related proteins (sFRPs) function as modulators of Wnt signaling through direct interaction with Wnts (33). We reported in our previous study that Wnt5ainduced RhoA activation and cell migration could be abolished by sFRP2 pretreatment in breast cancer cells (21). In the present study, we also found that sFRP2 blocked the Wnt5a-induced RhoA activation in glioblastoma cells. These results demonstrated that sFRP2 acted as an antagonist of Wnt5a mediating the progression of glioblastoma and breast cancer. A recent study in immunohistochemistry revealed that the expression level of soluble frizzled-related protein 3 (sFRP3) was decreased in the nucleus in higher grade astrocytoma, indicating the antagonistic ability of Wnt signaling (34). Further studies are needed to decipher whether sFRP2 and sFRP3 function in a common pathway or in parallel pathways to block Wnt5a signaling.

In conclusion, the present study partially clarified the associations between Wnt5a/RhoA signaling and glioblastoma progression. It is the first to demonstrate that Wnt5a may regulate the invasion of glioblastoma cells, at least in part via the Daam1/RhoA signaling pathway. Therefore, the Wnt5a/Daam1/RhoA signaling pathway is a candidate accelerator in glioblastoma and may be a potential clinical classification marker and therapeutic target for human glioblastoma.

\section{Acknowledgements}

The present study was supported by a grant from the National Natural Science Foundation of China (81472703) to Y.Z., a sponsorship of Jiangsu Overseas Research and Training Program for University Prominent Young and Middle-aged Teachers and Presidents to Y.Z., and a grant from the Joint Research Project of Southeast University and Nanjing Medical University (2242017K3DN41) to Y.Z.

\section{References}

1. Agnihotri S, Burrell KE, Wolf A, Jalali S, Hawkins C, Rutka JT and Zadeh G: Glioblastoma, a brief review of history, molecular genetics, animal models and novel therapeutic strategies. Arch Immunol Ther Exp 61: 25-41, 2013.

2. Rahman M, Abbatematteo J, De Leo EK, Kubilis PS, Vaziri S, Bova F, Sayour E, Mitchell D and Quinones-Hinojosa A: The effects of new or worsened postoperative neurological deficits on survival of patients with glioblastoma. J Neurosurg 127: 123-131, 2017.

3. Konar SK, Bir SC, Maiti TK, Patra DP, DiPoto Brahmbhatt AC Jacobsohn JA and Nanda A: Early dural metastasis from a case of glioblastoma with primitive neuroectodermal differentiation: A case report and literature review. J Clin Neurosci 35: 78-81, 2017.

4. Zhan T, Rindtorff $\mathrm{N}$ and Boutros $\mathrm{M}$ : Wnt signaling in cancer. Oncogene 36: 1461-1473, 2017.

5. Bienz M: Bio-sketch for oncogene review issue on Wnt signalling. Oncogene 25: 7441, 2006.

6. Widelitz R: Wnt signaling through canonical and non-canonical pathways: Recent progress. Growth Factors 23: 111-116, 2005.

7. Xiao YF, Yong X, Tang B, Qin Y, Zhang JW, Zhang D, Xie R and Yang SM: Notch and Wnt signaling pathway in cancer: Crucial role and potential therapeutic targets (Review). Int J Oncol 48: 437-449, 2016.

8. Tao H, Guo L, Chen L, Qiao G, Meng X, Xu B and Ye W: MSX1 inhibits cell migration and invasion through regulating the Wnt/ $\beta$-catenin pathway in glioblastoma. Tumour Biol 37: 1097-1104, 2016.

9. Dong Z, Zhou L, Han N, Zhang M and Lyu X: Wnt/ $\beta$-catenin pathway involvement in ionizing radiation-induced invasion of U87 glioblastoma cells. Strahlenther Onkol 191: 672-680, 2015.

10. Habas R, Kato Y and He X: Wnt/Frizzled activation of Rho regulates vertebrate gastrulation and requires a novel Formin homology protein Daam1. Cell 107: 843-854, 2001.

11. Witze ES, Litman ES, Argast GM, Moon RT and Ahn NG: Wnt5a control of cell polarity and directional movement by polarized redistribution of adhesion receptors. Science 320: 365-369, 2008

12. Lu C, Wang X, Zhu H, Feng J, Ni S and Huang J: Over-expression of ROR2 and Wnt5a cooperatively correlates with unfavorable prognosis in patients with non-small cell lung cancer. Oncotarget 6: 24912-24921, 2015.

13. Santos A, Bakker AD, de Blieck-Hogervorst JM and KleinNulend J: WNT5A induces osteogenic differentiation of human adipose stem cells via rho-associated kinase ROCK. Cytotherapy 12: 924-932, 2010.

14. Yu JM, Jun ES, Jung JS, Suh SY, Han JY, Kim JY, Kim KW and Jung JS: Role of Wnt5a in the proliferation of human glioblastoma cells. Cancer Lett 257: 172-181, 2007.

15. Hu B, Wang Q, Wang YA, Hua S, Sauvé CG, Ong D, Lan ZD, Chang Q, Ho YW, Monasterio MM, et al: Epigenetic activation of WNT5A drives glioblastoma stem cell differentiation and invasive growth. Cell 167: 1281-1295.e18, 2016.

16. Binda E, Visioli A, Giani F, Trivieri N, Palumbo O, Restelli S, Dezi F, Mazza T, Fusilli C, Legnani F, et al: Wnt5a drives an invasive phenotype in human glioblastoma stem-like cells. Cancer Res 77: 996-1007, 2017.

17. Louis DN, Ohgaki H, Wiestler OD, Cavenee WK, Burger PC, Jouvet A, Scheithauer BW and Kleihues P: The 2007 WHO classification of tumours of the central nervous system. Acta Neuropathol 114: 97-109, 2007.

18. Louis DN, Perry A, Reifenberger G, von Deimling A, Figarella-Branger D, Cavenee WK, Ohgaki H, Wiestler OD, Kleihues P and Ellison DW: The 2016 World Health Organization Classification of Tumors of the Central Nervous System: A summary. Acta Neuropathol 131: 803-820, 2016.

19. Pontén J and Macintyre EH: Long term culture of normal and neoplastic human glia. Acta Pathol Microbiol Scand 74: 465-486, 1968.

20. Allen M, Bjerke M, Edlund H, Nelander S and Westermark B: Origin of the U87MG glioma cell line: Good news and bad news. Sci Transl Med 8: 354re3, 2016.

21. Zhu Y, Tian Y, Du J, Hu Z, Yang L, Liu J and Gu L: Dvl2-dependent activation of Daam1 and RhoA regulates Wnt5a-induced breast cancer cell migration. PLoS One 7: e37823, 2012.

22. Lu M, Wang T, He M, Cheng W, Yan T, Huang Z, Zhang L, Zhang H, Zhu W, Zhu Y, et al: Tumor suppressor role of miR-3622b-5p in ERBB2-positive cancer. Oncotarget 8: 23008-23019, 2017. 
23. Zoico E, Darra E, Rizzatti V, Budui S, Franceschetti G, Mazzali G, Rossi AP, Fantin F, Menegazzi M, Cinti S, et al: Adipocytes WNT5a mediated dedifferentiation: A possible target in pancreatic cancer microenvironment. Oncotarget 7: 20223-20235, 2016.

24. Bitler BG, Nicodemus JP, Li H, Cai Q, Wu H, Hua X, Li T, Birrer MJ, Godwin AK, Cairns P, et al: Wnt5a suppresses epithelial ovarian cancer by promoting cellular senescence. Cancer Res 71: 6184-6194, 2011

25. Huang CL, Liu D, Nakano J, Ishikawa S, Kontani K, Yokomise H and Ueno M: Wnt5a expression is associated with the tumor proliferation and the stromal vascular endothelial growth factor - an expression in non-small-cell lung cancer. J Clin Oncol 23: 8765-8773, 2005.

26. Lee GT, Kang DI, Ha YS, Jung YS, Chung J, Min K, Kim TH, Moon KH, Chung JM, Lee DH, et al: Prostate cancer bone metastases acquire resistance to androgen deprivation via WNT5Amediated BMP-6 induction. Br J Cancer 110: 1634-1644, 2014.

27. Wu J and Mlodzik M: Wnt/PCP instructions for cilia in left-right asymmetry. Dev Cell 40: 423-424, 2017.

28. Pan Y, Guo X, Yang Z, Chen S, Lei Y, Lin M, Wang L, Feng C and Ke Z: AEG-1 activates Wnt/PCP signaling to promote metastasis in tongue squamous cell carcinoma. Oncotarget 7: 2093-2104, 2016.

29. López-Escobar B, Cano DA, Rojas A, de Felipe B, Palma F, Sánchez-Alcázar JA, Henderson D and Ybot-González P: The effect of maternal diabetes on the Wnt-PCP pathway during embryogenesis as reflected in the developing mouse eye. Dis Model Mech 8: 157-168, 2015.
30. Zhu Y, Shen T, Liu J, Zheng J, Zhang Y, Xu R, Sun C, Du J, Chen $Y$ and Gu L: Rab35 is required for Wnt5a/Dvl2-induced Rac1 activation and cell migration in MCF-7 breast cancer cells. Cell Signal 25: 1075-1085, 2013.

31. Liu J, Zhang Y, Xu R, Du J, Hu Z, Yang L, Chen Y, Zhu Y and $\mathrm{Gu}$ L: PI3K/Akt-dependent phosphorylation of GSK3 $\beta$ and activation of RhoA regulate Wnt5a-induced gastric cancer cell migration. Cell Signal 25: 447-456, 2013.

32. Liu W, Sato A, Khadka D, Bharti R, Diaz H, Runnels LW and Habas R: Mechanism of activation of the Formin protein Daam1. Proc Natl Acad Sci USA 105: 210-215, 2008.

33. Jones SE and Jomary C: Secreted Frizzled-related proteins: Searching for relationships and patterns. BioEssays 24: 811-820, 2002.

34. Pećina-Šlaus N, Kafka A, Varošanec AM, Marković L, Krsnik Ž, Njirić N and Mrak G: Expression patterns of Wnt signaling component, secreted frizzled-related protein 3 in astrocytoma and glioblastoma. Mol Med Rep 13: 4245-4251, 2016.

This work is licensed under a Creative Commons Attribution-NonCommercial-NoDerivatives 4.0 International (CC BY-NC-ND 4.0) License. 